\title{
A new algorithm for solving some mechanical problems
}

\author{
G.W. Wei * \\ Department of Computational Science, National University of Singapore, Singapore 117543, Singapore
}

\begin{abstract}
This paper explores the utility of a discrete singular convolution algorithm for solving certain mechanical problems. Benchmark mechanical systems, including plate vibrations and incompressible flows, are employed to illustrate the robustness and to test accuracy of the present algorithm. Numerical results indicate that the present approach is very accurate, efficient and reliable for solving the aforementioned problems. (c) 2001 Elsevier Science B.V. All rights reserved.
\end{abstract}

\section{Introduction}

Since many practical problems in science and engineering are either extremely difficult or impossible to solve by conventional analytical methods, numerical simulations play a more and more important role in handling these problems. The advent of high-performance computers has given tremendous impetus to all numerical methods for solving science and engineering problems. Although there has been a great deal of achievement in developing accurate, efficient and robust computational methods, finding numerical solutions for partial differential equations (PDEs) is still a challenge owing to the presence of possible singularities and/or homoclinic manifolds that induce sharp transitions in the solutions. The presence of these phenomena can be extremely sensitive to numerical algorithms and can easily lead to numerically induced spatial and/or temporal chaos [1]. The conventional approaches to these problems may be classified as either global methods [2-6] or local methods [7-16]. Global methods are highly localized in their spectral representations, but are unlocalized in the coordinate representation. By contrast, local methods have high spatial localization, but are delocalized in their spectral representations. Moreover, the use of global methods is usually restricted to structured grids, whereas, local methods can be implemented to blockstructured grids and even unstructured grids. In general, global methods are much more accurate than local methods, while the major advantages of local methods are their flexibility in handling complex geometries and boundary conditions. In ordinary applications, it is relatively safe and efficient to use either a global method or a local one for numerically solving an ordinary differential equation or a partial differential equation. However, when a differential equation has singularities and/or homoclinic orbits, neither the global methods nor the local methods can be applied without numerical instabilities. The global methods lose their accuracy near the singularities due to local high frequency components. The local methods have to be implemented in an adaptive manner, which greatly limits their accuracy and requires extremely small (spatial and/or temporal) mesh sizes. In many situations, the rate of convergence of a numerical method simply cannot match the divergent rate of the problem under study near a singularity. It is desirable to have a method that has both spectral and spatial localization, and is thus locally smooth and asymptotically

\footnotetext{
${ }^{*}$ Tel.: +65-874-6589; fax: +65-774-6756.

E-mail address: cscweigw@nus.edu.sg (G.W. Wei).
} 
decaying in both spectral and coordinate spaces. Particularly, such a method has the feature that combines global methods' accuracy with local methods' flexibility.

The discrete singular convolution (DSC) algorithm [17] was proposed as a potential approach for numerical realization of Hilbert transform, Abel transform, Radon transform, and delta transform. These transforms are essential to many practical applications, such as computational electromagnetics, computed tomography, molecular potential surface generation and dynamic simulation. The DSC algorithm has been tested for its applications to stochastic process analysis [17], nanoscale pattern formation of complex systems [18], homoclinic orbit of the Sine-Gordon singularity [19], and quantum eigenvalue problem of the Schrödinger equation [20]. The underlying mathematical structure for the DSC algorithm is the theory of distributions [21].

The purpose of this paper is to explore the utility and test the reliability of the DSC algorithm for mechanical applications. To this end, we consider two types of problems, plate vibrations and incompressible flows. This paper is organized as follows: The DSC algorithm is reviewed in Section 2. Some relevant parts of the algorithm are described in a greater detail than the original paper. Vibration analysis by the DSC algorithm is presented in Section 3. Eigenfunctions and eigenvalues of a rectangular plate and a circular plate are studied. Section 4 is devoted to fluid flow applications. We consider two test examples, the Taylor problem and a double shear layer flow, to illustrate the accuracy and robustness of the DSC approach for flow simulations. This paper ends with a conclusion.

\section{Theory and algorithm}

Singular convolutions are essential to many science and engineering problems, such as electromagnetics, Hilbert transform, Abel and Radon transforms. DSC is a general approach for the numerical realization of singular convolutions. By appropriate construction or approximation of a singular kernel, the discrete singular convolution can be an extremely efficient, accurate and reliable algorithm for practical applications [17].

It is most convenient to discuss singular convolution in the context of distributions. We denote $T$ a distribution and $\eta(t)$ an element of the space of test functions. A singular convolution can be expressed as

$$
F(t)=\int_{-\infty}^{\infty} T(t-x) \eta(x) \mathrm{d} x
$$

Here $T(t-x)$ is a singular kernel. Depending on the form of the kernel $T$, the singular convolution is the central issue for many science and engineering problems. For example, singular kernels of the Hilbert type have a general form of

$$
T(x)=\frac{1}{x^{n}}, \quad(n>0) .
$$

Here, kernels $T(x)=1 / x^{a},(0<a<1)$ define the Abel transform which is closely connected with a generalization of the tautochrone problem. Kernel $T(x)=1 / x$ commonly occurs in theory of linear response, signal processing, theory of analytic functions, and the Hilbert transform. Its three-dimensional version is important to the theory of electromagnetics. $T(x)=1 / x^{2}$ is the kernel used in tomography. Other interesting examples are singular kernels of the delta type

$$
T(x)=\delta^{(n)}(x), \quad(n=0,1,2, \ldots) .
$$

Here, kernel $T(x)=\delta(x)$ is important for interpolation of surfaces and curves, and $T(x)=\delta^{(n)}(x),(n=1,2, \ldots)$ are essential for numerically solving differential equations. However, a common feature of these kernels is that they are singular, i.e., they cannot be directly digitized in computers. In this regard, the singular convolution, (1), is of little numerical merit. To avoid the difficulty of using singular expressions directly in computers, sequences of approximations $\left(T_{\alpha}\right)$ of the distribution $T$ can be constructed 


$$
\lim _{\alpha \rightarrow \alpha_{0}} T_{\alpha}(x) \rightarrow T(x)
$$

where $\alpha_{0}$ is a generalized limit. Obviously, in the case of $T(x)=\delta(x)$, the sequence, $T_{\alpha}(x)$, is a delta sequence. Furthermore, with a good approximation, it makes sense to consider a discrete version

$$
F_{\alpha}(t)=\sum_{k} T_{\alpha}\left(t-x_{k}\right) f\left(x_{k}\right),
$$

where $F_{\alpha}(t)$ is an approximation to $F(t)$ and $\left\{x_{k}\right\}$ is an appropriate set of discrete points on which the discrete convolution (5) is well-defined. It is this discrete expression that makes a computer realization possible. Note that, the original test function $\eta(x)$ has been replaced by $f(x)$. The mathematical property or requirement of $f(x)$ is determined by the approximate kernel $T_{\alpha}$. In general, the convolution is required being Lebesgue integrable.

It is helpful to illustrate the algorithm by examples. A simple example is Shannon's kernel, $(\sin \alpha x) / \pi x$. Shannon's kernels are a delta sequence and thus provide an approximation to the delta distribution

$$
\lim _{\alpha \rightarrow \infty}\left\langle\frac{\sin \alpha x}{\pi x}, \eta(x)\right\rangle=\eta(0) .
$$

Other important examples include the Dirichlet kernel

$$
\frac{\sin \left[\left(l+\frac{1}{2}\right)\left(x-x^{\prime}\right)\right]}{2 \pi \sin \left[\frac{1}{2}\left(x-x^{\prime}\right)\right]},
$$

the modified Dirichlet kernel

$$
\frac{\sin \left[\left(l+\frac{1}{2}\right)\left(x-x^{\prime}\right)\right]}{2 \pi \tan \left[\frac{1}{2}\left(x-x^{\prime}\right)\right]},
$$

and the de la Vallée Poussin kernel

$$
\frac{1}{\pi \alpha} \frac{\cos \left[\alpha\left(x-x^{\prime}\right)\right]-\cos \left[2 \alpha\left(x-x^{\prime}\right)\right]}{\left(x-x^{\prime}\right)^{2}} .
$$

For sequences of the delta type, an interpolating (or quasi-interpolating) algorithm sampling at Nyquist frequency, $\alpha=\pi / \Delta$, has great advantage over a non-interpolating discretization. Therefore, the Shannon's kernel is discretized as

$$
\frac{\sin \left[\alpha\left(x-x^{\prime}\right)\right]}{\pi\left(x-x^{\prime}\right)} \rightarrow \frac{\sin \frac{\pi}{4}\left(x-x_{k}\right)}{\frac{\pi}{4}\left(x-x_{k}\right)} .
$$

In fact, not only the interpolating (or quasi-interpolating) nature guarantees the highest accuracy on the set of grid points, but also it provides the highest possible computational efficiency off a grid. This is because the Nyquist interval given by $[-\pi / \Delta, \pi / \Delta]$ is the largest possible sampling interval that is free of alias whenever an $L^{2}$ function $f(x)$ under study satisfies the Nyquist condition

$$
\operatorname{supp} \hat{f}(k) \subset\left\{-\frac{\pi}{\Delta}, \frac{\pi}{\Delta}\right\} \text {. }
$$

This fact can be formally addressed by Shannon's sampling theorem

$$
f(x)=\sum_{k=-\infty}^{\infty} f\left(x_{k}\right) \frac{\sin \frac{\pi}{4}\left(x-x_{k}\right)}{\frac{\pi}{4}\left(x-x_{k}\right)} .
$$

The significance of Shannon's sampling theorem is that by a discrete, but infinite set of sampling data, $\left\{f\left(x_{k}\right)\right\}$, one can actually recover a bandlimited $L^{2}$ function on a real line. Such bandlimited $L^{2}$ functions are known as elements of the Paley-Wiener reproducing kernel Hilbert space. The discrete Shannon's kernels, 
$\left\{\sin \frac{\pi}{4}\left(x-x_{k}\right) / \frac{\pi}{4}\left(x-x_{k}\right)\right\}_{k \in Z}$, are a complete set of sampling basis. Shannon's sampling theorem has great impact on information theory, signal and image processing because the Fourier transform of Shannon's kernel is an ideal low-pass filter for signals bandlimited to $[-\pi / \Delta, \pi / \Delta]$.

It is noted that the sequence of approximation can be improved by a regularizer $[23,24]$

$$
\lim _{\sigma \rightarrow \infty} R_{\sigma}(x)=1 .
$$

The regularizer is designed to increase the regularity of convolution kernels. For the delta sequence, it follows from Eq. (4) that

$$
\int \lim _{\alpha \rightarrow \alpha_{0}} T_{\alpha}(x) R_{\sigma}(x) \mathrm{d} x=R_{\sigma}(0)=1,
$$

where $R_{\sigma}(0)=1$ is the special requirement for a delta regularizer. A typical delta regularizer used in this work and elsewhere [24] is $\exp \left(-x^{2} / 2 \sigma^{2}\right)$. Therefore, Shannon's kernel is regularized as

$$
\frac{\sin (\pi / \Delta)\left(x-x_{k}\right)}{(\pi / \Delta)\left(x-x_{k}\right)} \rightarrow \frac{\sin (\pi / \Delta)\left(x-x_{k}\right)}{(\pi / \Delta)\left(x-x_{k}\right)} \mathrm{e}^{-\left(\left(x-x_{k}\right)^{2} / 2 \sigma^{2}\right)} .
$$

Since $\exp \left(-x^{2} / 2 \sigma^{2}\right)$ is a Schwartz class function, it makes the regularized kernel applicable to tempered distributions. Numerically, the regularized expression performs much better than Shannon's kernel for being used in a local approach for solving partial differential equations [24]. Qian and Wei [22] have recently given a rigorous error estimation of the regularized formulae.

The uniform, Nyquist rate, interpolating discretization and the regularization are also adopted for the Dirichlet kernel

$$
\frac{\sin \left[\left(l+\frac{1}{2}\right)\left(x-x^{\prime}\right)\right]}{2 \pi \sin \left[\frac{1}{2}\left(x-x^{\prime}\right)\right]} \rightarrow \frac{\sin \left(\frac{\pi}{4}\left(x-x_{k}\right)\right)}{(2 M+1) \sin \left(\frac{\pi}{4} \frac{x-x_{k}}{2 M+1}\right)} \exp \left(-\frac{\left(x-x_{k}\right)^{2}}{2 \sigma^{2}}\right) .
$$

In comparison to Shannon's kernel, the Dirichlet kernel has one more parameter $M$ which can be optimized to achieve better results in computations. Usually, we set a sufficiently large $M$ for various numerical applications. Obviously, the Dirichlet kernel converts to Shannon's kernel at the limit of $M \rightarrow \infty$. The uniform interpolating discretization and the regularization will also be used for the modified Dirichlet kernel

$$
\frac{\sin \left[\left(l+\frac{1}{2}\right)\left(x-x^{\prime}\right)\right]}{2 \pi \tan \left[\frac{1}{2}\left(x-x^{\prime}\right)\right]} \rightarrow \frac{\sin \left(\frac{\pi}{4}\left(x-x_{k}\right)\right)}{(2 M+1) \tan \left(\frac{\pi}{4} \frac{x-x_{k}}{2 M+1}\right)} \exp \left(-\frac{\left(x-x_{k}\right)^{2}}{2 \sigma^{2}}\right),
$$

and for the de la Vallée Poussin kernel

$$
\frac{1}{\pi \alpha} \frac{\cos \left[\alpha\left(x-x^{\prime}\right)\right]-\cos \left[2 \alpha\left(x-x^{\prime}\right)\right]}{\left(x-x^{\prime}\right)^{2}} \rightarrow \frac{2}{3} \frac{\cos \frac{\pi}{4}\left(x-x_{k}\right)-\cos \frac{2 \pi}{4}\left(x-x_{k}\right)}{\left[\frac{\pi}{4}\left(x-x_{k}\right)\right]^{2}} \exp \left(-\frac{\left(x-x_{k}\right)^{2}}{2 \sigma^{2}}\right),
$$

where $\bar{\Delta}=(3 / 2) \Delta$. Since $\pi / \Delta$ is proportional to the highest frequency which can be reached in the Fourier representation, the $\Delta$ should be very small for a given problem involving highly oscillatory functions or very high frequency components.

We use a symmetrically (or antisymmetrically) truncated singular kernel

$$
f^{(n)}(x) \approx \sum_{k=-W}^{W} \delta_{\alpha, \sigma}^{(n)}\left(x-x_{k}\right) f\left(x_{k}\right), \quad(n=0,1,2, \ldots),
$$

where $2 W+1$ is the computational bandwidth, or effective kernel support, which is usually smaller than the whole computational domain, $[a, b]$. Here $\delta_{\alpha, \sigma}^{(n)}\left(x-x_{k}\right)$ is a collective symbol for the $n$th derivative of any of 
the right-hand side of Eqs. (15)-(18). Note that kernel (19) is translationally invariant in the computational domain. Therefore, it is very simple to implement.

Qian and Wei [22] have recently provided a mathematical estimation for the choice of $W, \sigma$ and $\Delta$. For example, if the $L_{2}$ error for approximating an $L^{2}$ function $f$ is set to $10^{-\eta}(\eta>0)$, the following relations are to be satisfied

$$
r(\pi-B \Delta)>\sqrt{4.61 \eta}, \quad \text { and } \frac{W}{r}>\sqrt{4.61 \eta},
$$

where $r=\sigma / \Delta$ and $B$ is the frequency bound for the function of interest, $f$. The first inequality states that for a given grid size $\Delta$, a large $r$ is required for approximating high frequency component of an $L^{2}$ function. The second inequality indicates that if one chooses the ratio $r=3$, then the half bandwidth $W \sim 30$ can be used to ensure the highest accuracy in a double precision computation $(\eta=15)$. This theoretical estimation is in very good agreement with a previous numerical test [24].

Consider an operator $\mathcal{O}$ having a differential part $\mathscr{D}$ and a function part $F$

$$
\mathcal{O}=\mathscr{D}+F .
$$

In the DSC approach, it is convenient to choose a grid representation for the coordinate so that the function part $F$ of the operator is diagonal. Hence, its discretization is simply given by a direct interpolation on the grid

$$
F(x) \rightarrow F\left(x_{k}\right) \delta_{m, k} .
$$

The differential part of the operator on the coordinate grid is then represented by functional derivatives

$$
\mathscr{D}=\sum_{n} \mathrm{~d}_{n}(x) \frac{\mathrm{d}^{n}}{\mathrm{~d} x^{n}} \rightarrow \sum_{n} \mathrm{~d}_{n}\left(x_{m}\right) \delta_{\alpha, \sigma}^{(n)}\left(x_{m}-x_{k}\right)
$$

where $\mathrm{d}_{n}(x)$ is a coefficient and $\delta_{\alpha, \sigma}^{(n)}\left(x_{m}-x_{k}\right)$ is analytically given by

$$
\delta_{\alpha, \sigma}^{(n)}\left(x_{m}-x_{k}\right)=\left[\left(\frac{\mathrm{d}}{\mathrm{d} x}\right)^{n} \delta_{\alpha, \sigma}\left(x-x_{k}\right)\right]_{x=x_{m}} .
$$

Here $\delta_{\alpha, \sigma}\left(x-x_{k}\right)$ is a collective symbol for the right-hand sides of Eqs. (15)-(18). The differentiations in Eq. (24) can be easily carried out for a given $\delta_{\alpha, \sigma}\left(x-x_{k}\right)$. For example, if

$$
\delta_{\frac{\pi}{4}, \sigma}\left(x-x_{k}\right)=\frac{\sin \frac{\pi}{4}\left(x-x_{k}\right)}{(\pi / \Delta)\left(x-x_{k}\right)} \mathrm{e}^{-\left(x-x_{k}\right)^{2} / 2 \sigma^{2}},
$$

we have for $x \neq x_{k}[24]$

$$
\begin{aligned}
\delta_{\pi / \Delta, \sigma}^{(1)}\left(x_{m}-x_{k}\right)= & \frac{\cos \frac{\pi}{4}\left(x-x_{k}\right)}{\left(x-x_{k}\right)} \exp \left(-\frac{\left(x-x_{k}\right)^{2}}{2 \sigma^{2}}\right)-\frac{\sin \frac{\pi}{4}\left(x-x_{k}\right)}{\frac{\pi}{4}\left(x-x_{k}\right)^{2}} \exp \left(-\frac{\left(x-x_{k}\right)^{2}}{2 \sigma^{2}}\right) \\
& -\frac{\sin \frac{\pi}{4}\left(x-x_{k}\right)}{\frac{\pi}{4} \sigma^{2}} \exp \left(-\frac{\left(x-x_{k}\right)^{2}}{2 \sigma^{2}}\right), \\
\delta_{\pi / \Delta, \sigma}^{(2)}\left(x_{m}-x_{k}\right)= & -\frac{\frac{\pi}{4} \sin \frac{\pi}{4}\left(x-x_{k}\right)}{\left(x-x_{k}\right)} \exp \left(-\frac{\left(x-x_{k}\right)^{2}}{2 \sigma^{2}}\right)-2 \frac{\cos \frac{\pi}{4}\left(x-x_{k}\right)}{\left(x-x_{k}\right)^{2}} \exp \left(-\frac{\left(x-x_{k}\right)^{2}}{2 \sigma^{2}}\right) \\
& -2 \frac{\cos \frac{\pi}{4}\left(x-x_{k}\right)}{\sigma^{2}} \exp \left(-\frac{\left(x-x_{k}\right)^{2}}{2 \sigma^{2}}\right)+2 \frac{\sin \frac{\pi}{4}\left(x-x_{k}\right)}{\frac{\pi}{4}\left(x-x_{k}\right)^{3}} \exp \left(-\frac{\left(x-x_{k}\right)^{2}}{2 \sigma^{2}}\right) \\
& +\frac{\sin \frac{\pi}{4}\left(x-x_{k}\right)}{\frac{\pi}{4}\left(x-x_{k}\right) \sigma^{2}} \exp \left(-\frac{\left(x-x_{k}\right)^{2}}{2 \sigma^{2}}\right)+\frac{\sin ^{2} \frac{\pi}{4}\left(x-x_{k}\right)}{\frac{\pi}{4} \sigma^{4}}\left(x-x_{k}\right) \exp \left(-\frac{\left(x-x_{k}\right)^{2}}{2 \sigma^{2}}\right),
\end{aligned}
$$




$$
\begin{aligned}
\delta_{\pi / \Delta, \sigma}^{(3)}\left(x_{m}-x_{k}\right)= & -\frac{\frac{\pi^{2}}{\Delta^{2}} \cos \frac{\pi}{4}\left(x-x_{k}\right)}{\left(x-x_{k}\right)} \exp \left(-\frac{\left(x-x_{k}\right)^{2}}{2 \sigma^{2}}\right)+3 \frac{\frac{\pi}{\Delta} \sin \frac{\pi}{4}\left(x-x_{k}\right)}{\left(x-x_{k}\right)^{2}} \exp \left(-\frac{\left(x-x_{k}\right)^{2}}{2 \sigma^{2}}\right) \\
& +3 \frac{\frac{\pi}{4} \sin \frac{\pi}{4}\left(x-x_{k}\right)}{\sigma^{2}} \exp \left(-\frac{\left(x-x_{k}\right)^{2}}{2 \sigma^{2}}\right)+6 \frac{\cos \frac{\pi}{4}\left(x-x_{k}\right)}{\left(x-x_{k}\right)^{3}} \exp \left(-\frac{\left(x-x_{k}\right)^{2}}{2 \sigma^{2}}\right) \\
& +3 \frac{\cos \frac{\pi}{4}\left(x-x_{k}\right)}{\left(x-x_{k}\right) \sigma^{2}} \exp \left(-\frac{\left(x-x_{k}\right)^{2}}{2 \sigma^{2}}\right)+3 \frac{\left(x-x_{k}\right) \cos \frac{\pi}{4}\left(x-x_{k}\right)}{\sigma^{4}} \exp \left(-\frac{\left(x-x_{k}\right)^{2}}{2 \sigma^{2}}\right) \\
& -6 \frac{\sin \frac{\pi}{4}\left(x-x_{k}\right)}{\frac{\pi}{4}\left(x-x_{k}\right)^{4}} \exp \left(-\frac{\left(x-x_{k}\right)^{2}}{2 \sigma^{2}}\right)-3 \frac{\sin \frac{\pi}{4}\left(x-x_{k}\right)}{\frac{\pi}{4}\left(x-x_{k}\right)^{2} \sigma^{2}} \exp \left(-\frac{\left(x-x_{k}\right)^{2}}{2 \sigma^{2}}\right) \\
& -\frac{\left(x-x_{k}\right)^{2} \sin _{\frac{\pi}{4}}\left(x-x_{k}\right)}{\frac{\pi}{4} \sigma^{6}} \exp \left(-\frac{\left(x-x_{k}\right)^{2}}{2 \sigma^{2}}\right),
\end{aligned}
$$

and

$$
\begin{aligned}
& \delta_{\pi / \Delta, \sigma}^{(4)}\left(x_{m}-x_{k}\right)=4 \frac{\frac{\pi^{2}}{\Delta^{2}} \cos \frac{\pi}{\Delta}\left(x-x_{k}\right)}{\left(x-x_{k}\right)^{2}} \exp \left(-\frac{\left(x-x_{k}\right)^{2}}{2 \sigma^{2}}\right)+\frac{\frac{\pi^{3}}{\Delta^{3}} \sin \frac{\pi}{\Delta}\left(x-x_{k}\right)}{\left(x-x_{k}\right)} \exp \left(-\frac{\left(x-x_{k}\right)^{2}}{2 \sigma^{2}}\right) \\
& +4 \frac{\frac{\pi^{2}}{\Delta^{2}} \cos \frac{\pi}{4}\left(x-x_{k}\right)}{\sigma^{2}} \exp \left(-\frac{\left(x-x_{k}\right)^{2}}{2 \sigma^{2}}\right)-12 \frac{\frac{\pi}{\Delta} \sin \frac{\pi}{4}\left(x-x_{k}\right)}{\left(x-x_{k}\right)^{3}} \exp \left(-\frac{\left(x-x_{k}\right)^{2}}{2 \sigma^{2}}\right) \\
& -6 \frac{\frac{\pi}{4} \sin \frac{\pi}{4}\left(x-x_{k}\right)}{\left(x-x_{k}\right) \sigma^{2}} \exp \left(-\frac{\left(x-x_{k}\right)^{2}}{2 \sigma^{2}}\right)-6 \frac{\frac{\pi}{4}\left(x-x_{k}\right) \sin \frac{\pi}{4}\left(x-x_{k}\right)}{\sigma^{4}} \exp \left(-\frac{\left(x-x_{k}\right)^{2}}{2 \sigma^{2}}\right) \\
& -24 \frac{\cos \frac{\pi}{4}\left(x-x_{k}\right)}{\left(x-x_{k}\right)^{4}} \exp \left(-\frac{\left(x-x_{k}\right)^{2}}{2 \sigma^{2}}\right)-12 \frac{\cos \frac{\pi}{4}\left(x-x_{k}\right)}{\left(x-x_{k}\right)^{2} \sigma^{2}} \exp \left(-\frac{\left(x-x_{k}\right)^{2}}{2 \sigma^{2}}\right) \\
& -4 \frac{\left(x-x_{k}\right)^{2} \cos \frac{\pi}{4}\left(x-x_{k}\right)}{\sigma^{6}} \exp \left(-\frac{\left(x-x_{k}\right)^{2}}{2 \sigma^{2}}\right)+24 \frac{\sin \frac{\pi}{4}\left(x-x_{k}\right)}{\frac{\pi}{4}\left(x-x_{k}\right)^{5}} \exp \left(-\frac{\left(x-x_{k}\right)^{2}}{2 \sigma^{2}}\right) \\
& +12 \frac{\sin \frac{\pi}{4}\left(x-x_{k}\right)}{\frac{\pi}{4}\left(x-x_{k}\right)^{3} \sigma^{2}} \exp \left(-\frac{\left(x-x_{k}\right)^{2}}{2 \sigma^{2}}\right)+3 \frac{\sin \frac{\pi}{4}\left(x-x_{k}\right)}{\frac{\pi}{4}\left(x-x_{k}\right) \sigma^{4}} \exp \left(-\frac{\left(x-x_{k}\right)^{2}}{2 \sigma^{2}}\right) \\
& -2 \frac{\left(x-x_{k}\right) \sin \frac{\pi}{4}\left(x-x_{k}\right)}{\frac{\pi}{4} \sigma^{6}} \exp \left(-\frac{\left(x-x_{k}\right)^{2}}{2 \sigma^{2}}\right) \\
& +\frac{\left(x-x_{k}\right)^{3} \sin \frac{\pi}{4}\left(x-x_{k}\right)}{\frac{\pi}{4} \sigma^{8}} \exp \left(-\frac{\left(x-x_{k}\right)^{2}}{2 \sigma^{2}}\right) \text {. }
\end{aligned}
$$

At $x=x_{k}$, it is convenient to evaluate these derivatives separately

$$
\begin{aligned}
& \delta_{\pi / \Delta, \sigma}^{(1)}(0)=0, \\
& \delta_{\pi / \Delta, \sigma}^{(2)}(0)=-\frac{1}{3} \frac{3+\frac{\pi^{2}}{\Delta^{2}} \sigma^{2}}{\sigma^{2}}, \\
& \delta_{\pi / \Delta, \sigma}^{(3)}(0)=0,
\end{aligned}
$$

and

$$
\delta_{\pi / \Delta, \sigma}^{(4)}(0)=\frac{1}{5} \frac{15+10 \frac{\pi^{2}}{\Delta^{2}} \sigma^{2}+\frac{\pi^{4}}{\Delta^{4}} \sigma^{4}}{\sigma^{4}} .
$$


Note that the differentiation matrix in Eq. (23) is in general banded. This gives rise to great advantage in large scale computations. Extension to higher dimensions can be realized by tensorial products. We refer expression (24) and its higher dimensional generalizations as DSC matrices.

In the present study, we focus our attention to the regularized Shannon's kernel (15). Nevertheless, various other delta sequence kernels can be similarly employed [17]. The DSC parameters are chosen as $W=32$ and $\sigma / \Delta=3.8$ for the following computations except for specified ones. We select two classes of benchmark test problems, each from solid mechanics and fluid mechanics, to demonstrate the usefulness, to test the accuracy and to explore the limitations of the DSC algorithm. Details of these computations are described in the following two sections.

\section{Plate vibration}

Plate analysis is of great practical significance, associated with applications in many engineering fields, such as civil, mechanical, aerospace, etc. Apart from a few analytically solvable cases, there is no general solution for plate vibrations. Numerical simulation is one of the major approaches for plate analysis. Various numerical methods have been used for plate computations. These include the series expansion [25] for orthotropic plates, integral equation approach [26-28], methods of finite strips and finite elements [29-31], domain decomposition approaches [32,33], Galerkin methods [34], and Rayleigh-Ritz variational methods $[35,36]$, to name only a few. As our first attempt, we consider only isotropic plates with homogeneous boundary conditions for which analytical solutions are available for comparisons. Vibration analysis of plates with mixed boundary conditions and complex geometries will be accounted elsewhere.

Let us consider an isotropic plate with undeformed middle surface having a governing equation [37]

$$
\mathscr{L} w=\nabla^{4} w+\frac{N}{D} \nabla^{2} w-\frac{\rho \omega^{2}}{D} w=0
$$

where $w$ is solely a function of the spatial coordinates, $N$ the inplane force intensity, $D$ the flexural rigidity, $\rho$ the mass density per unit area, and $\omega$ the sinusoidal time response frequency. According to the accepted convention of the theory of elasticity, the normal force $N$ is positive in Eq. (33) if the plate is in compression and negative if the plate in tension.

\subsection{Rectangular plates}

The problem of all-side simply supported rectangular plates in absence of inplane forces $(N=0)$ is the simplest one to solve. The simply supported boundary conditions are given by

$$
\begin{aligned}
& w=0, \quad M_{x}=-D\left[\frac{\partial^{2} w}{\partial x^{2}}+v \frac{\partial^{2} w}{\partial y^{2}}\right]=0 \quad(\text { for } x=0, a) \\
& w=0, \quad M_{y}=-D\left[\frac{\partial^{2} w}{\partial y^{2}}+v \frac{\partial^{2} w}{\partial x^{2}}\right]=0 \quad(\text { for } x=0, b),
\end{aligned}
$$

where $v$ is the Poisson's ratio, $a$ and $b$ are the lengths of the rectangular plate. The analytical solution of this case is actually independent of the Poisson's ratio and is given by [37]

$$
w_{n m}=A_{n m} \sin \frac{n \pi x}{a} \sin \frac{m \pi y}{b}, \quad n, m=1,2, \ldots,
$$

where $A_{n m}$ is an amplitude coefficient determined from the initial condition of the problem, $n$ and $m$ are integers. The frequency is given by

$$
\omega_{n m}=\sqrt{\frac{D}{\rho}}\left[\left(\frac{n \pi}{a}\right)^{2}+\left(\frac{m \pi}{b}\right)^{2}\right] .
$$


Table 1

Errors for plate vibrations

\begin{tabular}{|c|c|c|c|c|c|c|c|c|}
\hline$N$ th & Exact $\times 10^{4}$ & Error & $N$ th & $\begin{array}{l}\text { Ex- } \\
\operatorname{act} \times 10^{4}\end{array}$ & Error & $N$ th & Exact $\times 10^{4}$ & Error \\
\hline 1 & 4 & $7.39(-13)$ & 34 & 2704 & $3.09(-11)$ & 67 & 9604 & $3.64(-12)$ \\
\hline 2 & 25 & $6.96(-14)$ & 35 & 2704 & $2.75(-12)$ & 68 & 10000 & $7.73(-13)$ \\
\hline 3 & 25 & $1.25(-12)$ & 36 & 2809 & $1.39(-12)$ & 69 & 10000 & $5.73(-12)$ \\
\hline 4 & 64 & $6.78(-13)$ & 37 & 2809 & $2.96(-11)$ & 70 & 10201 & $3.92(-12)$ \\
\hline 5 & 100 & $1.50(-13)$ & 38 & 3364 & $1.07(-13)$ & 71 & 10201 & $7.76(-13)$ \\
\hline 6 & 100 & $3.37(-13)$ & 39 & 3364 & $4.38(-13)$ & 72 & 10816 & $9.26(-14)$ \\
\hline 7 & 169 & $2.28(-13)$ & 40 & 3721 & $4.33(-13)$ & 73 & 10816 & $1.05(-12)$ \\
\hline 8 & 169 & $4.87(-13)$ & 41 & 3721 & $1.70(-13)$ & 74 & 11236 & $1.09(-12)$ \\
\hline 9 & 289 & $4.91(-13)$ & 42 & 4225 & $3.37(-14)$ & 75 & 11236 & $6.25(-13)$ \\
\hline 10 & 289 & $2.02(-13)$ & 43 & 4225 & $3.82(-13)$ & 76 & 11881 & $1.61(-12)$ \\
\hline 11 & 324 & $1.60(-12)$ & 44 & 4225 & $6.75(-13)$ & 77 & 11881 & $1.57(-13)$ \\
\hline 12 & 400 & $1.54(-14)$ & 45 & 4225 & $1.11(-12)$ & 78 & 12769 & $1.53(-13)$ \\
\hline 13 & 400 & $1.46(-12)$ & 46 & 4624 & $7.21(-13)$ & 79 & 12769 & $1.71(-13)$ \\
\hline 14 & 625 & $1.59(-12)$ & 47 & 4624 & $1.42(-13)$ & 80 & 13456 & $1.33(-12)$ \\
\hline 15 & 625 & $3.80(-13)$ & 48 & 5184 & $5.01(-12)$ & 81 & 13456 & $5.44(-12)$ \\
\hline 16 & 676 & $8.23(-14)$ & 49 & 5329 & $2.98(-12)$ & 82 & 13689 & $1.73(-12)$ \\
\hline 17 & 676 & $2.16(-12)$ & 50 & 5329 & $1.84(-13)$ & 83 & 13689 & $1.69(-13)$ \\
\hline 18 & 841 & $2.15(-12)$ & 51 & 5476 & $3.85(-13)$ & 84 & 14884 & $1.77(-12)$ \\
\hline 19 & 841 & $1.33(-12)$ & 52 & 5476 & $5.93(-13)$ & 85 & 14884 & $1.99(-12)$ \\
\hline 20 & 1024 & $5.00(-13)$ & 53 & 6400 & $8.27(-12)$ & 86 & 15625 & $1.35(-12)$ \\
\hline 21 & 1156 & $1.66(-13)$ & 54 & 6400 & $6.37(-11)$ & 87 & 15625 & $1.94(-12)$ \\
\hline 22 & 1156 & $5.99(-13)$ & 55 & 6724 & $6.36(-11)$ & 88 & 15625 & $3.41(-12)$ \\
\hline 23 & 1369 & $1.64(-13)$ & 56 & 6724 & $7.13(-12)$ & 89 & 15625 & $2.63(-11)$ \\
\hline 24 & 1369 & $7.93(-13)$ & 57 & 7225 & $3.59(-12)$ & 90 & 16384 & $2.54(-12)$ \\
\hline 25 & 1600 & $5.15(-12)$ & 58 & 7225 & $4.16(-13)$ & 91 & 16900 & $1.82(-11)$ \\
\hline 26 & 1600 & $5.53(-13)$ & 59 & 7225 & $4.38(-13)$ & 92 & 16900 & $6.87(-13)$ \\
\hline 27 & 1681 & $4.91(-13)$ & 60 & 7225 & $1.35(-12)$ & 93 & 16900 & $8.17(-12)$ \\
\hline 28 & 1681 & $3.49(-12)$ & 61 & 7921 & $4.27(-14)$ & 94 & 16900 & $1.27(-11)$ \\
\hline 29 & 2025 & $2.65(-13)$ & 62 & 7921 & $1.09(-12)$ & 95 & 18496 & $3.62(-14)$ \\
\hline 30 & 2025 & $7.40(-13)$ & 63 & 8100 & $5.52(-12)$ & 96 & 18496 & $3.89(-14)$ \\
\hline 31 & 2500 & $1.90(-12)$ & 64 & 8100 & $5.17(-14)$ & 97 & 18769 & $1.88(-12)$ \\
\hline 32 & 2500 & $1.13(-12)$ & 65 & 9409 & $1.90(-14)$ & 98 & 18769 & $2.73(-12)$ \\
\hline \multirow[t]{2}{*}{33} & 2500 & $8.20(-13)$ & 66 & 9409 & $7.10(-13)$ & 99 & 21025 & $2.00(-12)$ \\
\hline & & & & & & 100 & 21025 & $1.12(-11)$ \\
\hline
\end{tabular}

In the present computation, we use the DSC algorithm for the spatial discretization. The DSC-matrix for operator $\mathscr{L}$ is constructed according to Eq. (23). To simplify the problem further, we take the computational domain as $[0,10 \pi] \times[0,10 \pi]$ with 33 equally spaced grid points in each dimension. Results are obtained by a direct diagonalization of the DSC-biharmonic matrix by using a standard eigenvalue solver.

The first 100 exact eigenvalues and absolute errors of the present numerical calculations are listed in Table 1. With fewer than seven points per wavelength, some of the errors are as small as $10^{-14}$. It is evident that the present approach is extremely accurate for plate vibration analysis.

\subsection{Circular plates}

To illustrate the DSC approach further, we consider a circular plate with compressive inplane forces. It is well-known that since the direct diagonalization approach is limited by computer memory requirement, iterative methods are often used for large scale computations. However, iterative approaches are slightly more complicated to program and is subject to stability requirement. Instead of directly diagonalizing the DSC-operator matrix, it is useful to demonstrate the stability and the reliability of the present algorithm for iterative computations. To this end we introduce an artificial time dependence to the operator of Eq. (33) 


$$
\frac{\partial w}{\partial t}=\mathscr{L} w
$$

The steady state solution of Eq. (37) is the eigenfunction of the operator $\mathscr{L}$. In this case, we test a different boundary condition, the clamped boundary condition all around the circular plate

$$
w=0, \quad \frac{\partial w}{\partial r}=0 \quad(\text { for } r=R) .
$$

The solution of this problem has the form of [37]

$$
w(r, \theta)=\sum_{n, l, m}\left[A_{n, m} J_{m}\left(\zeta_{n, m} r / R\right)+B_{l, m} I_{m}\left(\eta_{l, m} r / R\right)\right] \mathrm{e}^{\mathrm{i} m \theta}+\mathrm{cc}, \quad m \geqslant 0, n, l>0,
$$
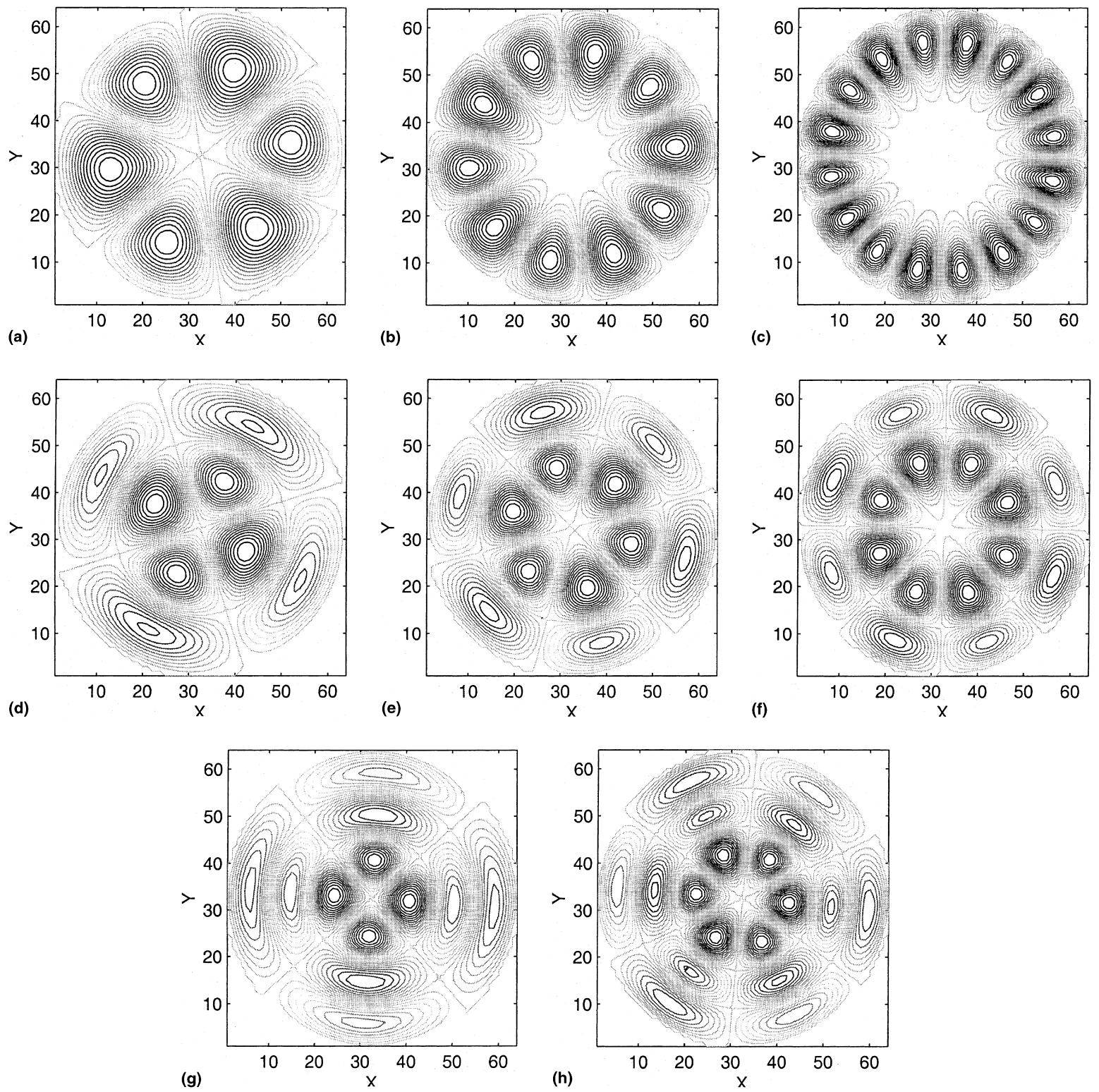

Fig. 1. Typical mode shapes of a clamped circular plate under compressive inplane forces. (a) mode $[1,3], \zeta_{1,3}=6.4, R_{1,3}=6.5 ;$ (b) mode $[1,5], \zeta_{1,5}=8.8, R_{1,5}=9.0$; (c) mode $[1,8], \zeta_{1,8}=12.2, R_{1,8}=12.3$; (d) mode $[2,2], \zeta_{2,2}=8.4, R_{2,2}=8.5 ;(\mathrm{e})$ mode $[2,3], \zeta_{2,3}=9.8$, $R_{2,3}=9.8$; (f) mode [2,4], $\zeta_{2,4}=11.1, R_{2,4}=11.1$; (g) mode [3,2], $\zeta_{3,2}=11.6, R_{3,2}=11.8 ;(\mathrm{h})$ mode $[3,3], \zeta_{3,3}=13.0, R_{3,3}=13.0$. 
where cc denotes the complex conjugate. Here $J_{m}\left(\zeta_{n, m} r / R\right)$ is the standard (cylinder) Bessel function, $I_{m}\left(\eta_{l, m} r / R\right)$ is the modified Bessel function of the first kind [37,38]. Here $\zeta_{n, m}$ and $\eta_{l, m}$ are nontrivial zeroes of $J_{m}$ and $I_{m}$, respectively. $A_{n, m}$ and $B_{l, m}$ are expansion coefficients.

In the present computation, we adapt the Crank-Nicolson scheme for time discretization. At $n$th iteration, the semi-discretized equation is

$$
\left(1-\frac{1}{2 \Delta t} \mathscr{L}\right) w^{n+1}=w^{n}+\frac{1}{2 \Delta t} \mathscr{L} w^{n}
$$

The DSC algorithm for spatial variable discretization is the same as prescribed in Eq. (23). For definiteness and simplicity, we consider only the case of $\omega=0, N=D$ in the present work. In such a case, the steady state solution of Eq. (37) is a superposition of the standard (cylinder) Bessel functions $J_{m}\left(\zeta_{n, m} r / R\right)$ for a given radius, $R$.

Eq. (37) is solved in polar coordinates $(r, \theta)$. A total number of 32 points are used for the $r$-grid and 64 for the $\theta$-grid. Note that the present approach is at its global limit for the $r$-grid, but is still a local algorithm for $\theta$-grid. The time increment is 1 for all simulations. The radius $R$ can be used as a control parameter to guide the system to an appropriate eigenstate. For simplicity we select only a few typical modes to demonstrate the ability of the present algorithm for handling circular geometry and clamped boundary condition. Fig. 1 illustrates the contour patterns of a few typical mode shapes characterized with their $[n, m]$ values. These modes are the steady states of Eq. (37) obtained by long time integrations. The present simulations are very stable and all results are very smooth. Remarkably, the control parameters, $R_{n, m}$, used for obtaining these states match well with the theoretical values [38], $\zeta_{n, m}$ (see the caption of Fig. 1).

\section{Incompressible flow}

Let us consider the incompressible Navier-Stokes and Euler equations

$$
\begin{aligned}
& u_{t}+u u_{x}+v u_{y}=-p_{x}+\frac{1}{R e}\left(u_{x x}+u_{y y}\right), \\
& v_{t}+u v_{x}+v v_{y}=-p_{y}+\frac{1}{R e}\left(v_{x x}+v_{y y}\right), \\
& u_{x}+v_{y}=0
\end{aligned}
$$

in a square $[0,2 \pi] \times[0,2 \pi]$ with periodic boundary conditions. Here $(u, v)$ is the velocity vector, $p$ is the pressure, $\operatorname{Re}(\operatorname{Re}>0)$ is the Reynolds number and $\operatorname{Re}=\infty$ defines the Euler equation. The NavierStokes equations are analytically solvable for appropriate initial values [40] and thus provide a benchmark test for potential numerical methods of fluid dynamics. With appropriate initial values, the Euler equation can be used to describe a flow field of vertically perturbed horizontal shear layers around a jet [39-41].

A simple time discretization as described by Yang and Shizgal [42] is chosen in the present DSC approach. At time $t_{n+1}$, there are three coupled equations for the velocity field

$$
\begin{aligned}
& \left(\frac{1}{R e} \nabla^{2}-\frac{1}{\Delta t}\right) u^{n+1}=p_{x}^{n+1 / 2}+S_{x}^{n}, \\
& \left(\frac{1}{R e} \nabla^{2}-\frac{1}{\Delta t}\right) v^{n+1}=p_{y}^{n+1 / 2}+S_{y}^{n},
\end{aligned}
$$

and for the pressure

$$
\nabla^{2} p^{n+1 / 2}=S_{p}^{n}
$$


Here $S_{x}^{n}, S_{y}^{n}$ and $S_{p}^{n}$ are

$$
\begin{aligned}
& S_{x}^{n}=-\frac{u^{n}}{\Delta t}+\left(u^{n} u_{x}^{n}+v^{n} u_{y}^{n}\right), \\
& S_{y}^{n}=-\frac{v^{n}}{\Delta t}+\left(u^{n} v_{x}^{n}+v^{n} v_{y}^{n}\right), \\
& S_{p}^{n}=\frac{1}{\Delta t}\left(u_{x}^{n}+v_{y}^{n}\right)-\left(u_{x}^{n}\right)^{2}-\left(v_{y}^{n}\right)^{2}-2 u_{y}^{n} v_{x}^{n} .
\end{aligned}
$$

At each time $t_{n+1}$, the pressure field $p^{n+1 / 2}$ is solved according to Eq. (46) from the known velocity field vector $\left(u^{n}, v^{n}\right)$. The velocity field vector $\left(u^{n+1}, v^{n+1}\right)$ is then updated according to Eqs. (44) and (45). All spatial differentiation matrices are constructed according to Eq. (23).

\subsection{The Navier-Stokes equation}

This example is used to illustrate the accuracy of the present approach. We take the initial condition as

$$
\begin{aligned}
& u(x, y, 0)=-\cos (x) \sin (y), \\
& v(x, y, 0)=\sin (x) \cos (y) .
\end{aligned}
$$

The exact solution for this case is given by:

$$
\begin{aligned}
& u(x, y, t)=-\cos (x) \sin (y) \mathrm{e}^{-(2 t / R e)}, \\
& v(x, y, t)=\sin (x) \cos (y) \mathrm{e}^{-(2 t / R e)}, \\
& p(x, y, t)=-\frac{1}{4}[\cos (2 x)+\cos (2 y)] \mathrm{e}^{-(4 t / R e)} .
\end{aligned}
$$

Our calculations are conducted at a variety of Reynolds numbers ranging from 100 to $\infty$. In each dimension, 31 equally spaced grid points $\left(N_{x}=N_{y}=31\right)$ are used for each dimension of the computational domain. The $W$ value is chosen as 30. The time mesh size is fixed as $0.001 . L_{2}$ and $L_{\infty}$ errors for various Reynolds numbers are listed in Table 2. Note that, the present result for the inviscid case $(R e=\infty)$ is about

\begin{tabular}{|c|c|c|c|c|c|c|c|c|}
\hline \multirow[t]{2}{*}{$R e$} & \multicolumn{2}{|l|}{$t=0.5$} & \multicolumn{2}{|l|}{$t=1.0$} & \multicolumn{2}{|l|}{$t=1.5$} & \multicolumn{2}{|l|}{$t=2.0$} \\
\hline & $L_{2}$ & $L_{\infty}$ & $L_{2}$ & $L_{\infty}$ & $L_{2}$ & $L_{\infty}$ & $L_{2}$ & $L_{\infty}$ \\
\hline $10^{2}$ & $3.2(-07)$ & $9.8(-08)$ & $6.4(-08)$ & $1.9(-07)$ & $9.4(-07)$ & $2.9(-07)$ & $1.2(-06)$ & $3.8(-07)$ \\
\hline $10^{3}$ & $3.2(-09)$ & $9.9(-10)$ & $6.5(-09)$ & $2.0(-09)$ & $9.7(-09)$ & $3.0(-09)$ & $1.3(-08)$ & $4.0(-09)$ \\
\hline $10^{4}$ & $3.2(-11)$ & $1.0(-11)$ & $6.4(-11)$ & $2.1(-11)$ & $9.7(-11)$ & $3.1(-11)$ & $1.3(-10)$ & $4.1(-11)$ \\
\hline $10^{5}$ & $7.1(-13)$ & $6.3(-13)$ & $1.5(-12)$ & $1.4(-12)$ & $2.3(-12)$ & $2.3(-12)$ & $3.3(-12)$ & $2.9(-12)$ \\
\hline$\infty$ & $9.8(-15)$ & $7.9(-15)$ & $1.5(-14)$ & $8.8(-15)$ & $2.3(-14)$ & $1.3(-14)$ & $3.3(-14)^{\mathrm{a}}$ & $1.8(-14)$ \\
\hline
\end{tabular}
$10^{10}$ times more accurate than that of $\mathrm{E}$ and $\mathrm{Shu}$ obtained by using a high order essentially non-oscillatory scheme [40]. It is evident that the accuracy of the DSC approach is extremely high, particularly when the Reynolds numbers are very large. Our other test calculations indicate that a better accuracy can be easily attained if the present low level time discretization is improved. Nevertheless, the present results are some of the best to our knowledge.

Table 2

$L_{2}$ and $L_{\infty}$ errors of the numerical solutions for the 2D Navier-Stokes equation

${ }^{\mathrm{a}} L_{2}=9.1(-04)$ was reported by $\mathrm{E}$ and Shu obtained by using a high order essentially non-oscillatory scheme [40]. 


\subsection{The Euler equation}

We now look at the case when $R e=\infty$ (the Euler equation) with sharply varying initial values. This example is chosen to illustrate the ability of the present algorithm for providing very fine resolution with a relatively coarse grid. The initial values are that of a jet in a doubly periodic geometry [39]

$$
\begin{aligned}
& u(x, y, 0)=\left\{\begin{array}{ll}
\tanh \left(\frac{2 y-\pi}{2 \rho}\right) & \text { if } y \leqslant \pi, \\
\tanh \left(\frac{3 \pi-2 y}{2 \rho}\right) & \text { if } y>\pi
\end{array}\right\}, \\
& v(x, y, 0)=\delta \sin (x),
\end{aligned}
$$

where $\delta=0.05$ is used for the present calculations. This initial value describes a flow field consisting of horizontal shear layers of finite thickness, perturbed by a small amplitude vertical velocity, making up the boundaries of the jet. The evolution under the Euler equation leads to a periodic array of large vortices, with the shear layer between the rolls being thinned by the large straining field. This problem is not analytically solvable and has been used to test numerical algorithms by many authors [39-41].

It is known that for certain choice of parameters the solution quickly develops into roll-ups with smaller and smaller scales, and the resolution is lost eventually with a fixed grid for local methods [40]. We choose a $65 \times 65$ grid for the computational domain. The results of different time units are plotted in Fig. 2. It is seen
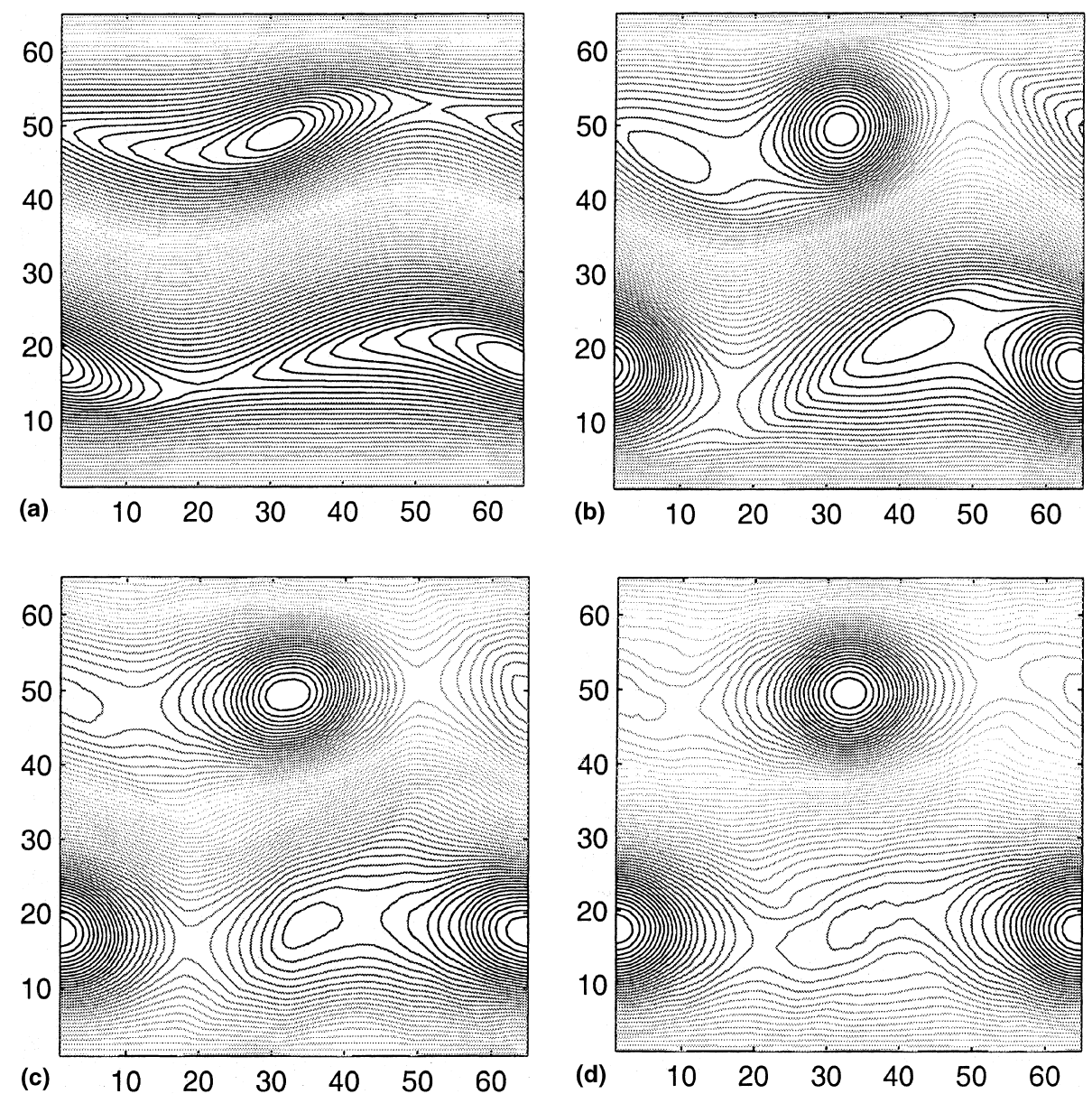

Fig. 2. The streamlines for the double shear layer problem integrated with $65^{2}$ grid points. (a) $t=4$; (b) $t=6$; (c) $t=8$; (d) $t=10$. 
that our solutions are smooth and stable for this case. We found that average kinetic energy is well preserved (a straight line) over the time history.

\section{Conclusion}

This paper explores the usefulness and tests the accuracy of a DSC [17] algorithm for mechanical applications. Two classes of benchmark examples are chosen to illustrate the present approach. The first class of problems is plate vibration analysis. Both a rectangular plate and a circular plate are employed to test the present approach. For the rectangular plate, a uniform, simply supported boundary condition is used and the biharmonic equation is analytically solvable. We use a direct matrix diagonalization method to calculate the first 100 eigenvalues. By using a reasonable mesh size we obtain results with errors as small as $10^{-14}$ for many eigenvalues. The second example considered is a circular plate with compressive inplane force and clamped boundary condition. The corresponding governing equation is also analytically solvable and thus it provides another benchmark test for numerical methods. To test the utility of the present approach for plate analysis, we choose an iterative scheme to extract plate mode shapes and vibration frequencies. A few typical results indicate that the present iterative-DSC algorithm works extremely well in predicting the shape and frequency of the circular plate vibration. In fact, the simulated modes match well with theoretical ones. Results of plates with mixed boundary conditions and complex geometries will be presented elsewhere.

In the second class of problems, we consider incompressible fluid flows. The first case in this class is the Navier-Stokes equation in a square box with periodic boundary condition, the Taylor problem. This problem admits an analytical solution. We use the DSC algorithm for spatial discretization and a first-order scheme for time advancing. Numerical simulations are performed over a wide range of Reynolds numbers with a small mesh. The present approach reaches the machine precision when the Reynolds numbers are sufficiently large. In particular, at $R e=\infty$, our result is $10^{10}$ times more accurate than that of $\mathrm{E}$ and Shu obtained by using a high-order essentially non-oscillatory scheme [40]. The DSC results are the best for this problem to our knowledge. The last case is the Euler equation with the initial value representing doubly periodic shear layer flows. To resolve small scale roll-ups produced in this problem is not an easy task, particularly with a relatively small mesh. Smooth and stable results are obtained in the present calculations. It is found that the kinetic energy of the system is preserved very well over the time history of the integration.

The illustrative calculations presented in this paper indicate that the present DSC approach is very accurate, efficient and reliable for certain mechanical applications. We note that although the DSC algorithm has a controllable accuracy for numerical approximations, it has, in general, a banded matrix. This is particularly important for large scale computations. Since the DSC matrix is banded, it has the potential to be implemented in complex geometries and mixed boundary conditions. These studies are in progress.

\section{Acknowledgements}

This work was supported in part by the National University of Singapore (Grant number: RP3982721).

\section{References}

[1] M.J. Ablowitz, B.M. Herbst, C. Schober, On the numerical solution of the Sine-Gordon equation, J. Comput. Phys. 126 (1996) 299-314.

[2] C. Lanczos, Trigonometric interpolation of empirical and analytical functions, J. Math. Phys. 17 (1938) $123-199$.

[3] J.W. Cooley, J.W. Tukey, An algorithm for the machine calculation of complex Fourier series, Math. Comput. 19 (1965) $297-301$.

[4] B.A. Finlayson, L.E. Scriven, The method of weighted residuals - a review, Appl. Mech. Rev. 19 (1966) $735-748$.

[5] S.A. Orszag, Comparison of pseudospectral and spectral approximations, Stud. Appl. Math. 51 (1972) $253-259$.

[6] B. Fornberg, On a Fourier method for the integration of hyperbolic equations, SIAM J. Numer. Anal. 12 (1975) $509-528$.

[7] G.E. Forsythe, W.R. Wasow, Finite-Difference Methods for Partial Differential Equations, Wiley, New York, 1960. 
[8] E. Isaacson, H.B. Keller, Analysis of Numerical Methods, Wiley, New York, 1966.

[9] O.C. Zienkiewicz, The Finite Element Method in Engineering Science, McGraw-Hill, London, 1971.

[10] C.S. Desai, J.F. Abel, Introduction to the Finite Element Methods, Van Nostrand Reinhold, New York, 1972.

[11] J.T. Oden, The Finite Elements of Non-linear Continua, McGraw-Hill, New York, 1972.

[12] B. Nath, Fundamentals of Finite Elements for Engineers, Athlone Press, London, 1974.

[13] R.T. Fenner, Finite Element Methods for Engineers, Imperial College Press, London, 1975.

[14] Y.K. Cheung, Finite Strip methods in Structural Analysis, Pergamon Press, Oxford, 1976.

[15] S.S. Rao, The Finite Element Method in Engineering, Pergamon Press, New York, 1982.

[16] J.N. Reddy, Energy and Variational Methods in Applied Mechanics, Wiley, New York, 1984.

[17] G.W. Wei, Discrete singular convolution for the Fokker-Planck equation, J. Chem. Phys. 110 (1999) $893-8942$.

[18] S. Guan, C.-H. Lai, G.W. Wei, Boundary controlled nanoscale morphology in a circular domain, Physica D, submitted, 1999.

[19] G.W. Wei, Discrete singular convolution method for the Sine-Gordon equation, Physica D 137 (2000) $247-259$.

[20] G.W. Wei, Solving quantum eigenvalue problems by discrete singular convolution, J. Phys. B 33 (2000) 343-359.

[21] L. Schwartz, Théore des Distributions, Hermann, Paris, 1951.

[22] L.W. Qian, G.W. Wei, A note on regularized Shannon's sampling formulae, J. Approx. Theory, submitted, 1999.

[23] G.W. Wei, D.S. Zhang, D.J. Kouri, D.K. Hoffman, Lagrange distributed approximating functionals, Phys. Rev. Lett. 79 (1997) $775-779$.

[24] G.W. Wei, Quasi-wavelets and quasi interpolating wavelets, Chem. Phys. Lett. 296 (1998) 215-222.

[25] Y. Narita, Application of a series-type method to vibration of orthotropic rectangular plates with mixed boundary conditions, J. Sound Vibr. 77 (1981) 345-355.

[26] C.C. Bartlett, The vibration and buckling of a circular plate clamped on part of its boundary and simply supported on the remainder, J. Mech. Appl. Math. 16 (1963) 431-440.

[27] L.M. Keer, B. Stahl, Eigenvalue problems of rectangular plates with mixed boundary conditions, J. Appl. Mech. 39 (1972) 513-520.

[28] Y. Hirano, K. Okazaki, Vibration of a circular plate having partly clamped or partly simply supported edges, Bull. Jpn Soc. Mech. Engrg. 19 (1976) 610-618.

[29] S.C. Fan, Y.K. Chueng, Flexural free vibrations of rectangular plates with complex supported conditions, J. Sound Vibr. 93 (1984) 81-94.

[30] F.E. Eastep, F.G. Hemming, Natural frequency of circular plates with partially free partially clamped edges, J. Sound Vibr. 84 (1982) 152-159.

[31] T. Mizusawa, T. Kaijita, Vibration and buckling of rectangular plates with non-uniform elastic constraints in rotation, Int. J. Solid Struct. 23 (1986) 45-55.

[32] K.M. Liew, K.C. Hung, K.Y. Lam, On the use of substructure method for vibration analysis of rectangular plates with discontinuous boundary conditions, J. Sound Vibr. 163 (1993) 451-462.

[33] K.M. Liew, K.C. Hung, M.K. Lim, On the use of domain decomposition method for vibration of symmetric laminates having discontinuities at the same edge, J. Sound Vibr. 178 (1994) 243-264.

[34] C.Y. Chia, Non-linear vibration anisotropic rectangular plates with non-uniform edge constraints, J. Sound Vibr. 101 (1985) $539-550$.

[35] A.W. Leissa, P.A.A. Laura, R.H. Gutierrez, Vibrations of rectangular plate with non-uniform elastic edge supports, J. Appl. Mech. 47 (1979) 281-292.

[36] K.M. Liew, C.M. Wang, Vibration analysis of plates by the pb2 Rayleigh-Ritz method: mixed boundary conditions reentrant corners and internal curved supports, Mech. Struct. Mech. 20 (1992) 281-292.

[37] A. Leissa, Vibration of Plates, Published for the Acoustical Society of America through the American Institute of Physics, 1993.

[38] G.N. Watson, Theory of Bessel functions, Cambridge, 1966.

[39] J.B. Bell, P. Colella, H.M. Glaz, A second-order projection method for the incompressible Navier-Stokes equations, J. Comput. Phys. 85 (1989) 257-283.

[40] W. E, C.W. Shu, A numerical resolution study of high-order essentially non-oscillatory schemes applied to incompressible flow, J. Comput. Phys. 110 (1994) 39-46.

[41] Z.J. Lou, R. Ferraro, A parallel incompressible flow solver package with a parallel multigrid elliptic kernel, J. Comput. Phys. 125 (1996) 225-243.

[42] H.H. Yang, B.R. Seymour, B.D. Shizgal, A Chebyshev pseudospectral multi-domain method for steady flow past a cylinder, Computers Fluids 23 (1994) 829-851. 